\title{
OPTIMIZATION OF DIGITAL CONTROL WITH DELAY BY PERIODIC VARIATION OF THE GAIN PARAMETERS
}

\author{
Tamas Insperger ${ }^{*, 1}$ Gabor Stepan* \\ * Budapest University of Technology and Economics, \\ Department of Applied Mechanics, Budapest, 1521, \\ Hungary
}

\begin{abstract}
The effect of periodic gain variation on the stability and robustness of digitally position controlled machines is investigated. Time delay and sampling effect of the control are included in the model. A discrete map is constructed that describes the dynamics of the digitally controlled system. The destabilizing effect of the time delay is shown by numerical examples. In order to improve stability properties, the control gains are varied according to the act and wait principle: they are switched between a constant value (act) and zero (wait). It is shown that the act and wait method leads to significant improvements in stability and also decreases the position error.
\end{abstract}

Keywords: PD controllers, gain modulation, optimal control, stability, time delay, sampling, dead-beat control

\section{INTRODUCTION}

Position control is a frequent mechanical controlling problem in robotics. The aim is to drive the robot arm into a desired position. Time delays in information transmission often arise in control systems, e.g., in remote control. This delay is constant and may vary from $10^{-6}$ to 1 second depending on the distance between the controlled robot and the controller, e.g. in master/slave systems. Consequently, this information delay is often negligible, but it may be crucial, for example, in space applications (see Vertut et al., 1976) or in systems controlled through the internet (Munir and Book, 2002). Caused by the delay of the control feedback, the governing equation is a delaydifferential equation (DDE). DDE's usually have infinite dimensional phase spaces (Hale and Lunel, 1993), therefore the linear stability conditions for the system parameters are complicated and often

\footnotetext{
1 E-mail: inspi@mm.bme.hu
}

do not have an analytical form. However, there exists several methods to analyze control systems with delayed feedback (Stepan, 1989, Just et al., 1999, Klein and Ramirez, 2001, Olgac and Sipahi, 2002, Asl and Ulsoy, 2002).

To achieve a clear picture about the behavior of the control, digital effects, like sampling, should also be included in the mechanical model. Sampling is a kind of delay in information transmission that often leads to unstable oscillations (Raibert and Craig, 1981, Craig, 1986, Stepan and Haller, 1995, Kollar et al., 2000)). Analytical investigation of simple models with 1 degree of freedom (DOF) play a central role in understanding technical phenomena and forming the common sense in design work (Insperger and Stepan, 2004).

Gain parameters and sampling time do not always provide stable motion and fast settling time, since these parameters are often hedged by other technical conditions. For these cases, periodic variation 
of the gains may stabilize or speed up the control (Khargonekar et al., 1985, Savkin and Petersen, 1998, Wolfe and Speyer, 2003). The idea of stabilizing by parametric excitation comes from the classical example of the pendulum: the upper position of a pendulum can be stabilized by vibrating its pivot point (Insperger, T., Horvath, R., 2000).

Due to the time periodic gains, the control system becomes time periodic. In this case, the infinite dimensional Floquet theory should be used for dynamic analysis. Even for simple time periodic delayed systems, like the delayed Mathieu equation, stability criteria have no closed form (Insperger and Stepan, 2002a). However, there exist several approximation techniques for analyzing time periodic delayed systems (Insperger and Stepan, 2002b, Bayly et al., 2003, Elbeyly and Sun, 2004, Butcher et al., 2004).

One effective way for analyzing periodic DDEs is the semi-discretization method (Insperger and Stepan, 2002b, Elbeyly and Sun, 2004). The point of the method is that only the delayed terms are discretized, the actual time domain terms are left in the original form, and the DDE is approximated by a series of ordinary differential equations (ODEs). The sampling effect of digital controls gives also a semi-discrete nature to the system: although, the system is delayed, the connection between states at discrete sampling instants can still be described by ODEs. This serves a finite dimensional discrete map model similarly to the semi-discretization method.

In this paper, the effect of periodic gain variation on the stability and robustness of digitally position controlled machines is investigated in the presence of time delays larger than the sampling time itself. The "act and wait" control technique is used: the gains are constant for the first sampling period (act), then they are zero for a certain number of samplings (wait), then they are constant again, etc. The effect of time delay and the periodic gains are investigated via stability charts and optimal controls are given. It is shown that the "act and wait" control technique improves the stability properties, and may lead to dead-beat control. Furthermore, the position error caused by the Coulomb friction can significantly decrease by this method.

\section{MECHANICAL MODEL, STABILITY AND ROBUSTNESS}

The 1 DOF mechanical model of position control is shown in Figure 1. The position of mass $m$ is sensed and a control force $Q$ is applied to push the mass into the desired zero position. The system is governed by the differential equation

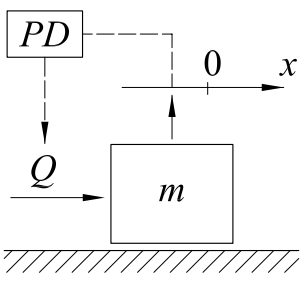

Fig. 1. Control model.

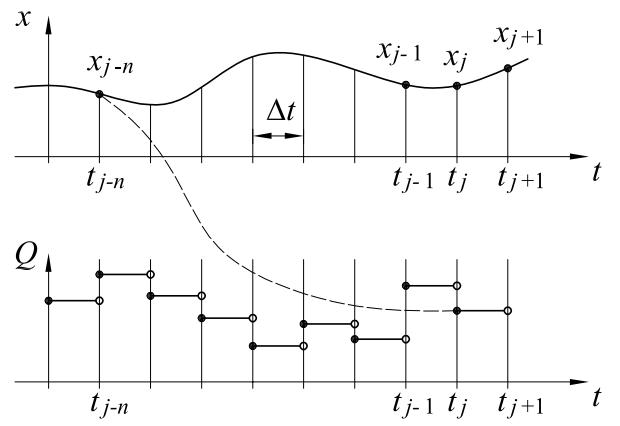

Fig. 2. Sampling effect with delay.

$$
m \ddot{x}(t)=Q\left(t, x_{t}, \dot{x}_{t}\right),
$$

where $x_{t}(\vartheta)=x(t+\vartheta)$ and $\dot{x}_{t}(\vartheta)=\dot{x}(t+\vartheta)$ with $\vartheta \in[-\sigma, 0]$ represent delay effects of the feedback.

Let the sampling time be $\Delta t$, and the delay of the control be $\tau=n \Delta t$, where integer $n$ is the delay parameter. The control force $Q$ is updated at each sampling instant $t_{j}=j \Delta t(j \in \mathbb{Z})$ by the delayed discrete values $x\left(t_{j-n}\right)$ and $\dot{x}\left(t_{j-n}\right)$ (see Figure 2$)$ :

$$
\begin{array}{r}
Q\left(t, x_{t}, \dot{x}_{t}\right)=-P_{j} x\left(t_{j-n}\right)-D_{j} \dot{x}\left(t_{j-n}\right), \\
t \in\left[t_{j}, t_{j+1}\right),
\end{array}
$$

where $P_{j+k}=P_{j}, D_{j+k}=D_{j}$ are the periodic control gains. Integer $k$ is the gain period parameter, that is, the gains are periodic with period $T=k \Delta t$. Now, the governing equation reads

$m \ddot{x}(t)=-P_{j} x\left(t_{j-n}\right)-D_{j} \dot{x}\left(t_{j-n}\right), \quad t \in\left[t_{j}, t_{j+1}\right)$.

Use the notations $x_{j}=x\left(t_{j}\right)$ and $\dot{x}_{j}=\dot{x}\left(t_{j}\right)$ for all $j \in \mathbb{Z}$. Stepwise integration of equation (3) gives the velocity and the displacement for the next sampling instant. Setting $t=t_{j+1}$ gives the following formula for two succeeding samples:

$$
\begin{aligned}
& \dot{x}_{j+1}=\dot{x}_{j}-\frac{P_{j} \Delta t}{m} x_{j-n}-\frac{D_{j} \Delta t}{m} \dot{x}_{j-n}, \\
& x_{j+1}=x_{j}+\dot{x}_{j} \Delta t-\frac{P_{j} \Delta t^{2}}{2 m} x_{j-n}-\frac{D_{j} \Delta t^{2}}{2 m} \dot{x}_{j-n} .
\end{aligned}
$$

Equations (4) and (5) can be represented as the discrete map

$$
\mathbf{y}_{j+1}=\mathbf{A}_{j} \mathbf{y}_{j}
$$

where $\mathbf{y}_{j}=\operatorname{col}\left(x_{j} \dot{x}_{j} x_{j-1} \dot{x}_{j-1} \ldots x_{j-n} \dot{x}_{j-n}\right)$ and the coefficient matrix is 

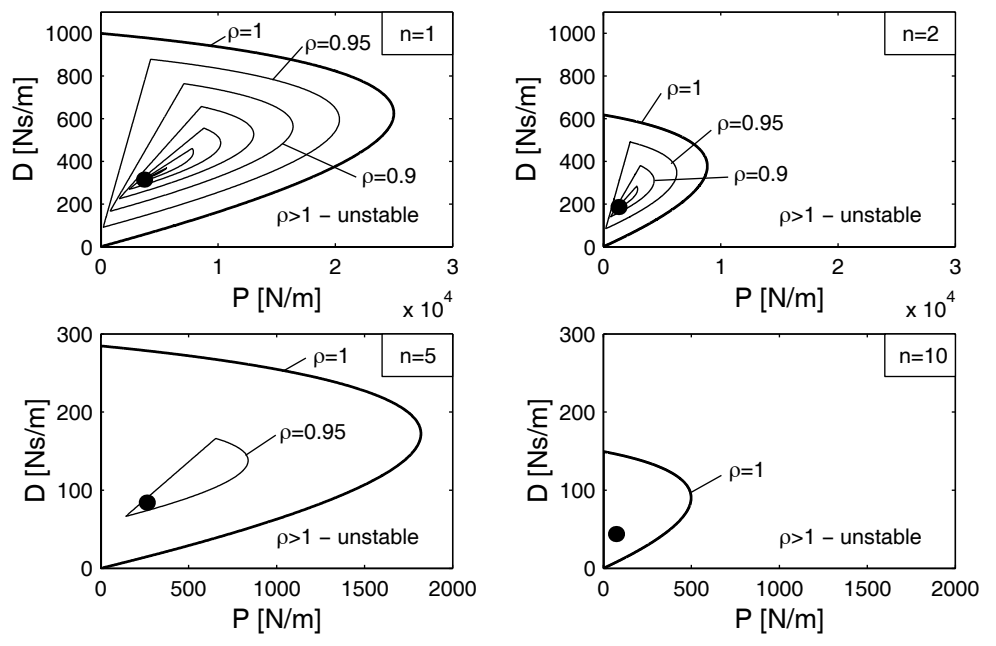

Fig. 3. Stability charts for different delay parameters $n$. Stability boundaries $(\rho=1)$ are denoted by thick lines.

$$
\mathbf{A}_{j}=\left(\begin{array}{ccccccccc}
1 & \Delta & 0 & 0 & \ldots & 0 & 0 & -\frac{P_{j} \Delta t^{2}}{2 m} & -\frac{D_{j} \Delta t^{2}}{2 m} \\
0 & 1 & 0 & 0 & \ldots & 0 & 0 & -\frac{P_{j} \Delta t}{m} & -\frac{D_{j} \Delta t}{m} \\
1 & 0 & 0 & 0 & \ldots & 0 & 0 & 0 & 0 \\
0 & 1 & 0 & 0 & \ldots & 0 & 0 & 0 & 0 \\
\vdots & \vdots & \vdots & \vdots & \ddots & \vdots & \vdots & \vdots & \vdots \\
0 & 0 & 0 & 0 & \ldots & 1 & 0 & 0 & 0 \\
0 & 0 & 0 & 0 & \ldots & 0 & 1 & 0 & 0
\end{array}\right) .
$$

Note, that the larger the delay parameter $n$ is, the larger the size of the discrete map is. For stability analysis, the Floquet transition matrix over the period $T=k \Delta t$ is determined by coupling the solutions:

$$
\boldsymbol{\Phi}=\mathbf{A}_{k-1} \mathbf{A}_{k-2} \ldots \mathbf{A}_{0} .
$$

Now, the stability of the control is determined by the eigenvalues of matrix $\boldsymbol{\Phi}$. If these eigenvalues are inside the unit disc of the complex plane, then the control is asymptotically stable (Kuo, 1977, Lakshmikantham and Trigiante, 1988). The critical (largest in modulus) eigenvalue $\mu_{1}$ determines the robustness of the control. For a stable control, the value $\left|\mu_{1}\right|$ characterizes the decrease of the error ratio over gain period $T: x_{j+k}<\left|\mu_{1}\right| x_{j}$. Introduce the decrease index $\rho=\left|\mu_{1}\right|^{1 / k}$ that characterizes the average error decrease ratio over the sampling period $\Delta t$. Controllers with different gain period parameters $k$ can be compared via the decrease index.

For the future investigations, the following mass and sampling time will be used: $m=10 \mathrm{~kg}$, $\Delta t=0.01 \mathrm{~s}$, while the delay parameter $n$ and gain period parameter $k$ will be changed. One aim during the control design is to find the optimal control parameters, i.e., the control gains, where the decrease ratio is minimal. In the presence of Coulomb friction force $C$, the position error is $\Delta=$ $C / P_{\max }$, where $P_{\max }=\max _{i}\left(P_{i}\right)$. Another aim of the control design is to decrease the position error, that is to increase $P_{\max }$.

\section{CONTROL WITH CONSTANT GAINS}

If the gain period parameter $k$ is set to 1 , then the gains are constant during the control. For this autonomous case, stability charts are presented in Figure 3 for different delay parameters $n$. The charts were determined via point-by-point evaluation of the transition matrix (8) over a $500 \times$ 500 sized grid, and a contour plot was used to obtain the transition curves associated to different decrease indices $\rho=1,0.95,0.9$, etc. Obviously, the stability boundaries are the transition curves where $\rho=1$.

It can be seen, that the larger the time delay is, the less the area of the stable domain is (note the different scales of the axes). The optimal gains, where the decrease index $\rho$ is minimal, were also determined numerically. These optimal gains are denoted by black points in Figure 3 and are also presented in Table 1. It can be seen, that the larger the time delay is, the smaller the optimal decrease index is, that is, the stability and the robustness of the control worsen with increasing feedback delay. Our numerical result is checked at $n=1$, where $\rho=2 / 3$ is an analytically proven value (Stepan et al., 1990).

It can also be observed in Figure 3 and in Table 1, that the optimal proportional gain decrease, that is, the position error increase with increasing delay parameter $n$.

Table 1. Optimal control gains and the corresponding decrease indices for different delay parameters $n$.

\begin{tabular}{|c|c|c|c|}
\hline$n$ & $P[\mathrm{~N} / \mathrm{m}]$ & $D[\mathrm{Ns} / \mathrm{m}]$ & $\rho$ \\
\hline 1 & 3706 & 314.8 & 0.6667 \\
\hline 2 & 1291 & 186.1 & 0.7887 \\
\hline 5 & 263.1 & 84.04 & 0.8987 \\
\hline 10 & 72.01 & 43.97 & 0.9457 \\
\hline
\end{tabular}



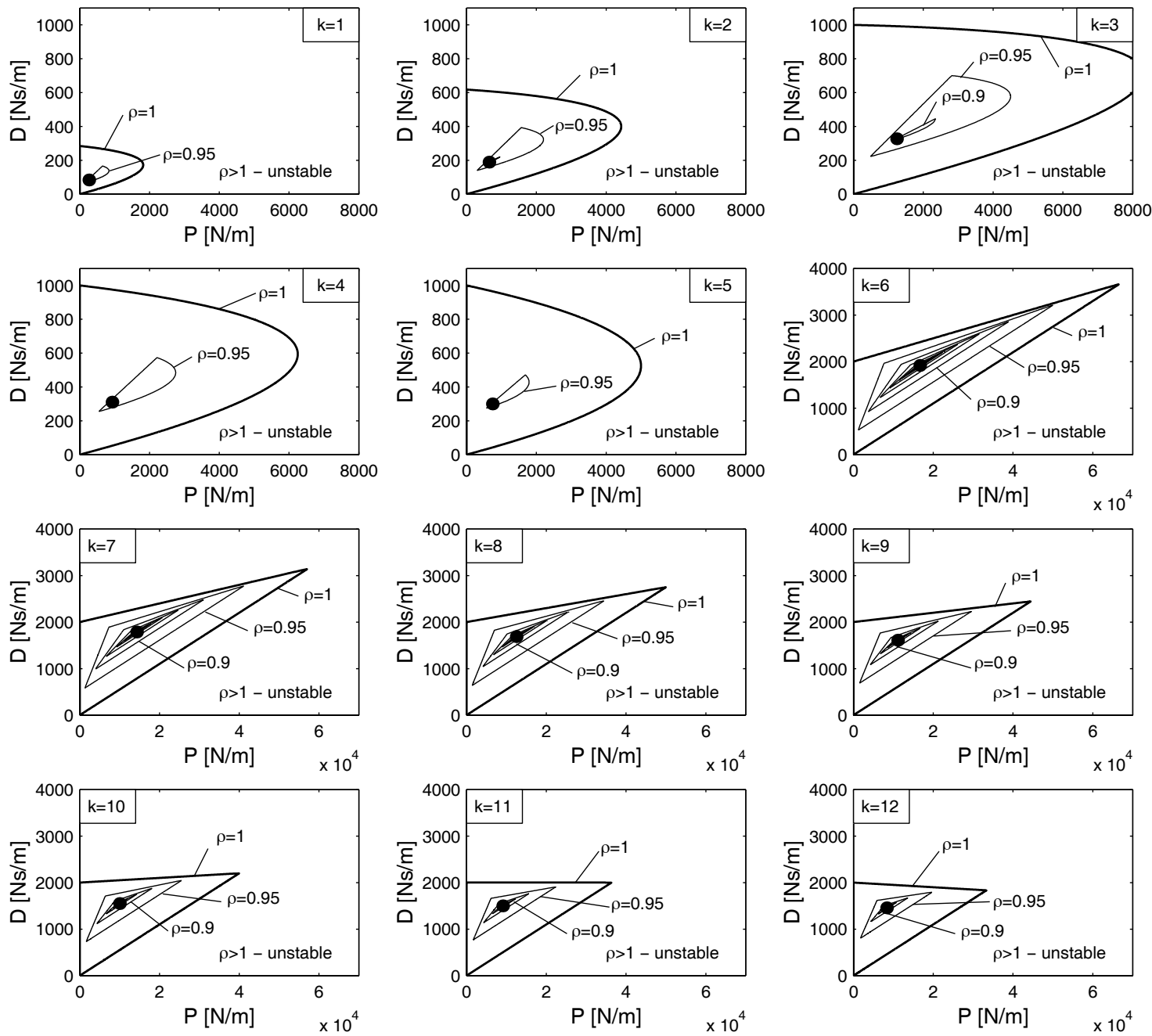

Fig. 4. Stability charts for delay parameter $n=5$ with different gain period parameters $k$. Stability boundaries $(\rho=1)$ are denoted by thick lines.

\section{CONTROL WITH PERIODICALLY VARYING GAINS}

Now, the gain parameters will be modified as

$$
P_{j}=g_{j} P, \quad D_{j}=D g_{j},
$$

where $g_{j}$ is a switching function:

$$
g_{j}=\left\{\begin{array}{lll}
1 & \text { if } t \in\left[t_{0}+h T, t_{1}+h T\right), & h \in \mathbb{Z} \\
0 & \text { if } t \in\left[t_{1}+h T, t_{k}+h T\right), & h \in \mathbb{Z}
\end{array}\right.
$$

The control is switched on for a sampling period $\left[t_{0}, t_{1}\right)$ and it is switched off for the remaining $k-1$ number of sampling intervals, i.e., for $\left[t_{1}, t_{k}\right)$. This is the act and wait principle of control systems with delayed feedback.

Stability charts for delay parameter $n=5$ and different gain period parameters $k$ are presented in Figure 4. The optimal gains with minimal robustness index are denoted by black points and are also presented in Table 2. For gain period parameters $k=1,2, \ldots, 5$, there are no significant changes in stability properties, only a slight decay of the optimal decrease index can be observed, while the optimal proportional gain increases definitely resulting smaller position error. In these cases, the duration of waiting (zero gains) is less than the time delay.

For the case $k=6$, there is a drastic change in the stability chart. The stable domain increases by a factor of 10 (note the scale of the axes) resulting

Table 2. Optimal control gains and the corresponding decrease index for delay parameters $n=5$ and different gain period parameters $k$.

\begin{tabular}{|c|c|c|c|}
\hline$k$ & $P[\mathrm{~N} / \mathrm{m}]$ & $D[\mathrm{Ns} / \mathrm{m}]$ & $\rho$ \\
\hline 1 & 263.1 & 84.04 & 0.8987 \\
\hline 2 & 645.3 & 189.3 & 0.8881 \\
\hline 3 & 1237 & 327.4 & 0.8736 \\
\hline 4 & 927.6 & 310.3 & 0.9036 \\
\hline 5 & 743.0 & 300.2 & 0.9222 \\
\hline 6 & 16667 & 1917 & 0 \\
\hline 7 & 14286 & 1785 & 0 \\
\hline 8 & 12500 & 1688 & 0 \\
\hline 9 & 11111 & 1611 & 0 \\
\hline 10 & 10000 & 1550 & 0 \\
\hline 11 & 9091 & 1500 & 0 \\
\hline 12 & 8333 & 1458 & 0 \\
\hline
\end{tabular}




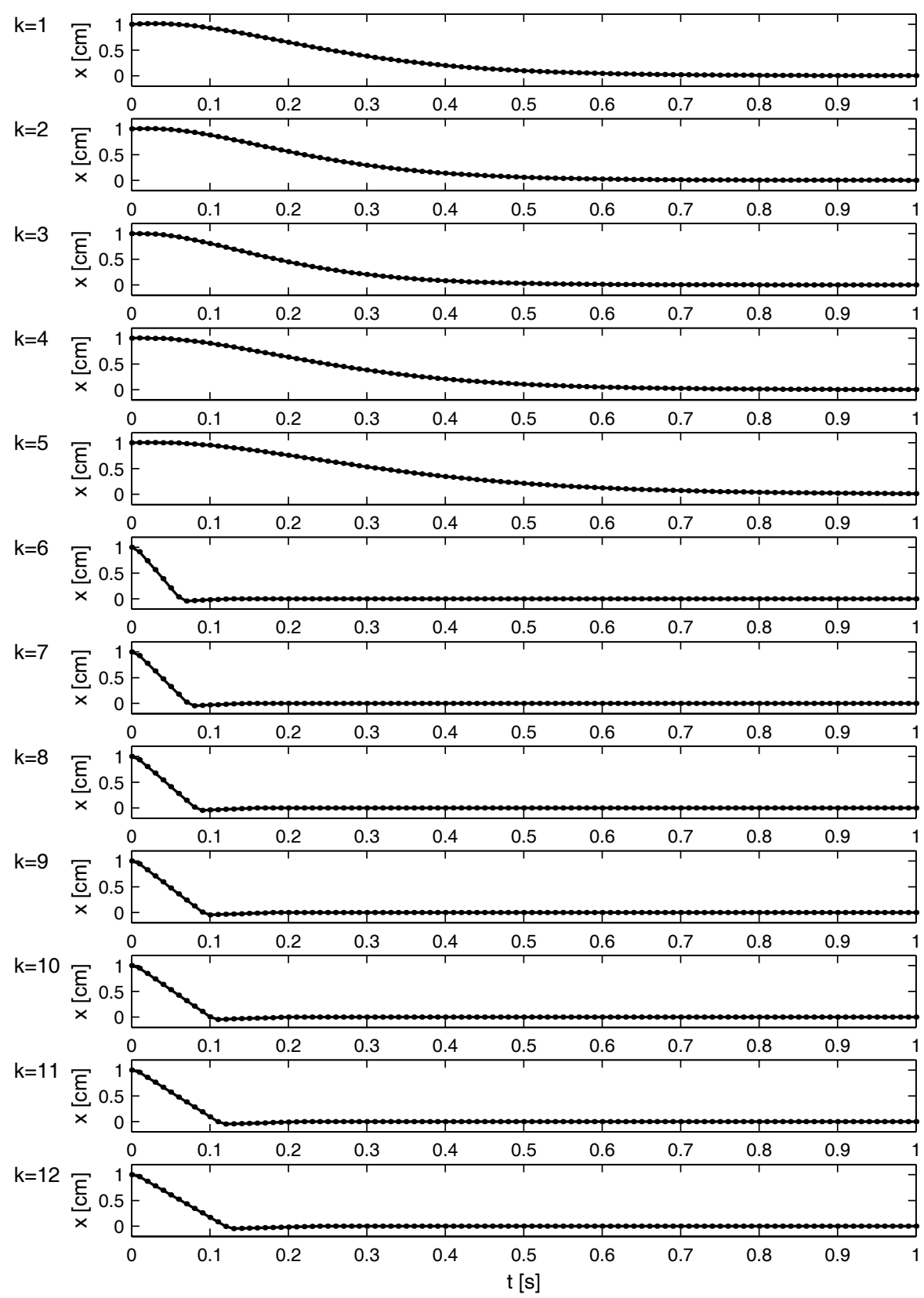

Fig. 5. Simulations for delay parameters $n=5$ and different gain period parameters $k$.

a significant improvement in position error, and the optimal control gains result in zero decrease index $(\rho=0)$, that is, in a dead-beat control. For $k>6$, the optimal control still results in deadbeat control, but the area of stable parameter domains gets slightly smaller. Also, the optimal proportional gains get smaller with increasing $k$ when $k>6$. This shows, that both the stability condition and the position error are optimal for the case $k=6$.

Numerical simulations were carried out according to equation (6) for different gain period parameters $k$ with the associated optimal gains given in Table 2. The simulation plots are shown in Figure 5 . As it was predicted by the decrease indices in Table 2, the best control was obtained for the case $k=6$.

\section{CONCLUSION}

The PD position control of a one degree of freedom system was investigated for the case when the gain parameters are varied periodically according to the act and wait principle. It was shown, that in spite of the destabilizing effect of the time delay, the control system can be optimized by properly chosen gain values and properly chosen gain variation period. A qualitative improvement in stability and position error was found for the case when the gain period parameter $k$ is just larger than the time delay parameter $n$. The results were confirmed by simulations. 


\section{ACKNOWLEDGMENT}

This research was supported in part by the Zoltán Magyary Postdoctoral Fellowship of Foundation for Hungarian Higher Education and Research, by the Hungarian National Science Foundation under grant no. OTKA F047318 and OTKA T043368.

\section{REFERENCES}

Asl, F. M., Ulsoy, A. G., 2002, Analysis of a system of linear delay differential equations, Journal of Dynamic Systems, Measurements and Control, 125(2), pp. 215-223.

Bayly, P. V., Halley, J. E., Mann, B. P., Davies, M. A., 2003, Stability of interrupted cutting by temporal finite element analysis, Journal of Manufacturing Science and Engineering, 125(2), pp. 220-225.

Butcher, E. A., Ma, H., Bueler, E., Averina, V., Szabo, Zs., 2004, Stability of time-periodic delay-differential equations via Chebyshev polynomials, International Journal for $\mathrm{Nu}$ merical Methods in Engineering, 59(7), pp. 895-922.

Craig, J. J., 1986, Introduction to Robot Mechanics and Control, Addison-Willey, Reading.

Elbeyly, O., Sun, J. Q., 2004, On the semidiscretization method for feedback control design of linear systems with time delay, Journal of Sound and Vibration, 273(1-2), pp. 429-440.

Hale, J. K., Lunel, S. M. V., 1993, Introduction to Functional Differential Equations, SpringerVerlag, New York.

Insperger, T., Horvath, R., 2000, Pendulum with harmonic variation of the suspension point, Periodica Polytechnica, 44(1), pp. 39-46.

Insperger, T., Stepan, G., 2002a, Stability chart for the delayed Mathieu equation, Proceedings of The Royal Society, Mathematical, Physical and Engineering Sciences, 458(2024), pp. 1989-1998.

Insperger T., Stepan, G., 2002b, Semi-Discretization Method for Delayed Systems, International Journal for Numerical Methods in Engineering, 55(5), pp. 503-518.

Insperger, T., Stepan, G., 2004, Stability improvements of robot control by periodic variation of the gain parameters, in Proceedings of the 11th World Congress in Mechanism and $M a-$ chine Science, Tianjin, China, China Machinery Press, pp. 1816-1820.

Just, W., Reibold, E., Benner, H., Kacperski, K., Fronczak, P., Holyst, J., 1999, Limits of time- delayed feedback control, Physics Letters A, 254, pp. 158-164.

Khargonekar, P., Poolla, K., Tannenbaum, A., 1985, Robust control of linear time invariant plants using periodic compensation, IEEE Transaction on Automatic Control, 30(11), pp. 1088-1095.

Klein, E. J., Ramirez, W. F., 2001, State controllability and optimal regulator control of time-delayed systems, International Journal of Control, 74(3), pp. 281-289.

Kollar, L. E., Stepan, G., Hogan, J. S., 2000, Sampling Delay and Backlash in Balancing Systems, Periodica Polytechnica, 44(1), pp. 77-84.

Kuo, B. C., 1977, Digital Control Systems, SRL Publishing Company, Champaign, Illinois.

Lakshmikantham, V., Trigiante, D., 1988, Theory of Difference Equations, Numerical Methods and Applications, Academic Press, London.

Munir, S., Book, W. J., 2002, Control techniques and programming issues for time delayed internet based teleoperation, Journal of Dynamic Systems, Measurements and Control, 125(2), pp. 205-214.

Olgac, N., Sipahi, R., 2002, An Exact Method for the Stability Analysis of Time-Delayed Linear Time-Invariant (LTI) Systems, IEEE Transactions on Automatic Control, 47(5), pp. 793-977.

Raibert, M. H., Craig, J. J., 1981, Hybrid position force control for computer controlled manipulators, ASME Journal of Dynamic Systems, Measurement and Control, 102, pp. 125-133.

Savkin, A. V., Petersen, I. R., 1998, Almost optimal LQ-control using stable periodic controllers, Automatica, 34(10), pp. 1251-1254.

Stepan, G., 1989, Retarded Dynamical Systems, Longman, Harlow.

Stepan, G., Steven A., Maunder, L., 1990, Design principles of digitally controlled robots, Mechanism and Machine Theory, 25, pp. 515-527.

Stepan, G., Haller, G., 1995, Quasiperiodic oscillations in robot dynamics, Nonlinear Dynamics, 8, pp. 513-528.

Vertut, J., Charles, J., Coiffet, P. and Petit. M., 1976, Advance of the new MA23 force reflecting manipulator system, Proceedings of the Second Symposium on Theory and Practice of Robots and Manipulators, Udine, Italy, pp. 50-57.

Wolfe, J. D., Speyer, J. L., 2003, The periodic optimality of LQ controllers satisfying strong stabilization, Automatica, 39, pp. 1735-1746. 